\title{
Expression Level of Serum FGF-21 in Patients with Hypothyroidism and Correlation Analysis of Biochemical Indexes
}

\author{
Li Chunhui ${ }^{1}$, Sun Kexin ${ }^{2}$, Wang Huiyan ${ }^{2^{*}}$ \\ ${ }^{1}$ Beihua University Affiliated Hospital Nuclear Medicine Discipline, Jilin, 132013, China. \\ ${ }^{2}$ Jinlin Medical College Test School, Jilin, 132013, China
}

\begin{abstract}
Objective: To explore the expression level and correlation analysis of fibroblast growth factor 21 (FGF-21) and its biochemical indexes in serum of patients with hypothyroidism. Method: To collect 168 cases of patients in the Affiliated Hospital of Beihua University, 62 cases of hyperthyroidism group, 52 cases of hypothyroidism group, 54 cases of healthy group, which are adopted respectively by enzyme-linked immunosorbent assay (ELISA) detection of FGF-21; chemiluminescence detection of thyroid stimulating hormone (TSH), serum free triiodothyronine (FT3), free thyroxine (FT4); detection of , biochemical analyzer of total cholesterol (TC) and triglyceride (TG), low density lipoprotein (LDL), as well as high density lipoprotein (HDL) and other indicators. Results: (1) The amount of FGF-21, TSH, TC, TG and LDL of serum in hypothyroidism group is significantly higher than that in control group $(\mathrm{P}<0.05)$, the amount of FT3, FT4 and HDL of serum in free thyroxine is significantly lower than that in control group $(\mathrm{P}<0.05)$. The amount of FGF-21, $\mathrm{TSH}, \mathrm{TC}, \mathrm{TG}$ and LDL of serum in hyperthyroidism group is significantly lower than that in control group $(\mathrm{P}<0.05)$, the amount of FT3, FT4 and HDL of serum in free thyroxine is significantly higher than that in control group $(\mathrm{P}<0.05)$; (2)Through the correlation analysis between plasma FGF-21, the level of plasma FGF-21 in patient with hypothyroidism is positively correlated with age, gender, TSH, TC, LDL. Conclusion: The level of serum FGF-21 is increased in hypothyroidism patients, which is correlated with TSH, TC, TG and LDL, etc. which may be the risk factors for thyroid dysfunction and result in the development of disease. The elevation of TC, TG and LDL in patients with hypothyroidism may also be one of the main causes of dyslipidemia.
\end{abstract}

Keywords: hypothyroidism; FGF-21; biochemical index; correlation

\section{Introduction}

Hypothyroidism (Hypothyroidism) is an endocrine disease characterized by Hypothyroidism, which is caused by the lack of secretion or lack of thyroid function. Thyroid hormone plays a role in liver, fat and other tissue organs, which can participate in the synthesis, decomposition and transport of cholesterol (TC) and triglyceride (TG). When the function of thyroid occurs disorder, it can be accompanied by the abnormal metabolism of fat, such as obesity, type II diabetes, hypertension, and lipid metabolism disorder and so on ${ }^{[1]}$. Fibroblast growth factor 21 (fibroblast growth factor 21, FGF-21) is a kind of new regulatory factor related to the glucolipid metabolism, which can increase the body's energy, with lower blood lipid, including three acyl glycerin and cholesterol, low density lipoprotein level, which also can enhance the intake of fat cells and inhibition of glucagon secretion and so on. Fgf-21 can improve the function of islet cells and promote glucose absorption in adipose tissue ${ }^{[2]}$. 
Hypothyroidism can affect blood lipid abnormalities, while serum FGF-21 is the regulation factor of lipid metabolism related to cytokines, in the course of disease, so as to explore what's the connection and correlation among them. Thus this study is to explore serum FGF-21 of patients with hypothyroidism and biochemical indexes of the expression level as well as its correlation analysis.

\section{Data and Method}

\section{Research object}

From July, 2015 to June,2016 06, collecting 62 cases in Affiliated Hospital of Beihua University who were diagnosed as hyperthyroidism group, among them, 26 cases are male, 36 cases are female, the average age is $(30 \pm 16) ; 52$ cases in hypothyroidism group, 22 cases are male, 30 cases are female, the average age is (32 \pm 2$)$; 54 cases in healthy group, 22 cases are male, 32 cases are female, the average age is $(36 \pm 14)$.

Detection of FGF-21

The level of human fibroblast growth factor 21 (FGF-21) can be detected by double antibody sandwich method. FGF-21 kit is purchased from Shanghai Bong Yi Biological Science Technology Co., Ltd. Adopting the standard instrument to determine the absorbance at the wavelength of $450 \mathrm{~nm}$ by enzyme (OD), according to the standard curve, to calculate the concentration of fibroblast growth factor 21 in the test sample (FGF-21) .

Detection of TSH, FT3, T4

The chemiluminescence instrument from Bo Yang Biological Science and Technology Shanghai Co., Ltd. and its kit can be used for determination.

Detection of TC,TG,LDLand HDL

The above kits biochemical analyzer Backman AU5821 are provided by KELONG Shanghai.

\section{Statistical Method}

Adopting SPSS17.0 data processing software, which is lined with the normal distribution of data,expressed by mean \pm standard $(\mathrm{x} \pm)$. The data of non-normal distribution can be represented by four bits distance, and the median can be converted to normal data after natural logarithm. The two groups can be compared with two independent samples t to test. $\mathrm{P}<0.05$ as the difference, which has statistical significance.

\section{Results}

The level of TSH, FT3 and FT4 in serum free thyroxine compared with TSH in serum

hypothyroidism group is significantly higher than that in the control group $(\mathrm{P}<0.05)$, the amount of FT3 and FT4 in serum free thyroxine is significantly lower than that in the control group ( $\mathrm{P}<0.05)$, the serum TSH in hyperthyroid group is significantly lower than that in the control group $(\mathrm{P}<0.05)$, the amount of FT3 and FT4 of serum free thyroxine is significantly higher than that in the control group $(\mathrm{P}<0.05)$ (see Table 1). 
Table 1 Expression Level of Serum TSH, FT3 and FT4 $(\mathrm{x}+\mathrm{s})$

\begin{tabular}{lcccc}
\hline Grouping & The number of cases & TSH $(\mathrm{mIU} / \mathrm{L})$ & $\mathrm{FT} 3(\mathrm{pmoI} / \mathrm{L})$ & $\mathrm{FT} 4(\mathrm{pmoI} / \mathrm{L})$ \\
\hline Hyperthyroidism group & 52 & $42.23 \pm 29.78^{*}$ & $3.12 \pm 0.63^{*}$ & $5.58 \pm 0.78^{*}$ \\
GD group & 62 & $0.12 \pm 0.03^{*}$ & $20.78 \pm 10.86$ & $38.27 \pm 13.86^{*}$ \\
Health Group & 54 & $2.15 \pm 1.24$ & $5.23 \pm 0.61$ & $14.32 \pm 2.08$ \\
\hline \multicolumn{5}{c}{ Note: compared with the control group $* \mathrm{P}<0.05$}
\end{tabular}

The Comparison of Serum FGF-21, TC, TG, LDL and Index Level of HDL

The level of Serum FGF-21、TC、TG、LDL in hypothyroidism group is higher than that in the control group $(\mathrm{P}<0.05)$, while the level of serum FGF-21、TC、TG, LDL in the hyperthyroidism group is significantly lower than that in the control group $(\mathrm{P}<0.05)$ (See Table 2).

Table 2 Comparison of the indicator level of FGF-21, TC, TG, LDL and HDL ( $\mathrm{x} \pm \mathrm{s})$

\begin{tabular}{|c|c|c|c|c|c|}
\hline Grouping & FGF-21(ng/mL) & $\mathrm{TC}(\mathrm{mmol} / \mathrm{L})$ & $\mathrm{TG}(\mathrm{mmol} / \mathrm{L})$ & $\mathrm{LDL}(\mathrm{mmol} / \mathrm{L})$ & $\mathrm{HDL}(\mathrm{mmol} / \mathrm{L})$ \\
\hline Hyperthyroidism group $(n=52)$ & $30.23 \pm 0.12 *$ & $7.28 \pm 0.89$ & $3.34 \pm 1.13^{*}$ & $4.03 \pm 1.39$ & $0.86 \pm 0.32 *$ \\
\hline GD group $(n=62)$ & $0.16 \pm 0.02 *$ & $5.36 \pm 1.02$ & $1.16 \pm 0.08^{*}$ & $3.45 \pm 0.02 *$ & $1.58 \pm 0.49$ \\
\hline Health Group $(\mathrm{n}=54)$ & $1.82 \pm 0.14$ & $6.12 \pm 0.76^{*}$ & $2.24 \pm 0.81$ & $2.98 \pm 1.02$ & $1.34 \pm 0.25$ \\
\hline
\end{tabular}

Note: compared with the control group $* \mathrm{P}<0.05$

\section{Correlation Analysis of Plasma FGF-21}

The plasma FGF-21 level in patients with hypothyroidism is positively correlated with age $(\mathrm{r}=0.143, \mathrm{P}<0.05)$, gender $(\mathrm{r}=0.651, \mathrm{P}<0.05)$, TG $(\mathrm{r}=0.298, \mathrm{P}<0.05)$, TSH $(\mathrm{r}=0.746, \mathrm{P}<0.01)$, and TSH ( $\mathrm{r}=0.746)$. The level of serum FGF-21 in patients with hyperthyroidism is positively correlated with TSH $(\mathrm{r}=0.287, \mathrm{P}<0.05)$ and $\mathrm{TG}(\mathrm{r}=0.285, \mathrm{P}<0.05)$. The level of plasma FCF-21 in the control group is positively correlated with age $(r=0.259, \mathrm{P}<0.05)$, (Table 3$)$.

Table 3 Correlation Analysis on Plasma FGF-21 with Its Parameters

\begin{tabular}{|c|c|c|c|c|c|c|c|c|c|}
\hline Grouping & Age & Gender & $\mathrm{TSH}$ & FT3 & FT4 & $\mathrm{TC}$ & $\mathrm{TG}$ & LDL & HDL \\
\hline \multicolumn{10}{|l|}{ Hyperthyroidism group } \\
\hline The value of $\mathrm{r}$ & 0.143 & 0.082 & 0.746 & .233 & $0.214 \quad 0$ & 0.3160 . & .298 & 0.346 & $0.021 *$ \\
\hline The value of $\mathrm{P}$ & $0.035^{*}$ & $0.651^{*}$ & $0.000 * * 0$. & .068 & $0.248 \quad 0 .($ & $.023 * 0.04$ & $47 * 0$. & $.048^{*}$ & .864 \\
\hline \multicolumn{10}{|l|}{ GD group } \\
\hline The value of $r$ & 0.124 & 0.090 & 0.287 & 0.112 & 0.106 & 0.1620 & 0.285 & 0.191 & 0.147 \\
\hline The value of $P$ & 0.423 & 0.536 & $0.024^{*}$ & 0.840 & 0.803 & 0.381 & $0.035^{*}$ & 0.184 & 0.242 \\
\hline \multicolumn{10}{|l|}{ Health Group } \\
\hline The value of $r$ & 0.259 & 0.189 & 0.163 & 0.074 & 0.115 & $0.002 *$ & 0.068 & 0.187 & 0.035 \\
\hline The value of $P$ & $0.023^{*}$ & 0.156 & 0.184 & 0.540 & $40 \quad 0.526$ & $6 \quad 0.891$ & 0.554 & 0.423 & 0.721 \\
\hline
\end{tabular}




\section{Discussion}

FGF-21 is a hormone like fibroblast growth factor. It has many functions such as growth, repair, metabolism and so on. It is a new glucose and lipid metabolism regulating factor, which is mainly expressed in the liver ${ }^{[3]}$. In diabetic obesity animal model,we find out that fibroblast growth factor 21 can interfere and improve blood glucose and blood lipid level, which is closely related to blood glucose and lipid metabolism ${ }^{[4]}$. In starvation, the expression of FGF-21 in mRNA can be detected in mice, and the concentration of FGF-21 in serum is increased at the same time. The effect may depend on the regulation of PPARa ${ }^{[5]}$. Studies have shown that FGF-21 is significantly increased in endocrine and metabolic diseases, renal failure dialysis patients, polycystic ovary syndrome and other diseases, which is reduced in anorexia nervosa ${ }^{[6]}$.

Hypothyroidism is a disease of the thyroid itself, which causes the synthesis, storage and secretion of thyroid hormones and disorders of metabolism in the body. When Mason ${ }^{[7]}$ as well as other people made study on hypothyroidism and hyperthyroidism serum cholesterol level, through blood lipid, 295 cases of primary hypothyroidism patients showed us the results that $91.5 \%$ patients are abnormal blood lipid, about $1.5 \%$ are belong ti type IV hyperlipidemia, only about $8.5 \%$ of the blood lipid patients are within normal range. The result of this study shows: the level of serum TC, TG, LDL in hypothyroidism group is higher than that in the control group $(\mathrm{P}<0.05)$, which shows that the serum lipid composition of hypothyroidism patients occurred abnormal changes of thyroid hormone, which may cause upregulation of the activity of HMGR (3-hydroxy-3-methyl-glutaryl-coenzyme A reductase), increasing its synthesis at the same time, so as to promote the synthesis of TC, while it will reduce TC when it occurs the synthesis of hypothyroidism, the total effect of hypothyroidism is TC, TG as well as the increase of LDL-C ${ }^{[8]}$. Adams ${ }^{[9]}$ and other people speculated that the expression of FGF-21 may be regulated by thyroxine, and its mediated metabolic changes are similar to that of FGF-21. In this study, serum FGF-21 level can be detected in hyperthyroidism group and healthy group. It was found that serum FGF-21 level in hyperthyroidism group was significantly lower than that in hypothyroidism group, and serum FGF-21 level in hypothyroidism group was significantly higher. The results showed that patients with hypothyroidism due to thyroid hormone deficiency, basal metabolic rate, protein synthesis and decomposition slowed down at the same time, renal blood flow, glomerular filtration rate and renal tubular re-absorption and secretion were decreased, renal excretion of water and the reduction of hydrophilic substances accumulate in the tissues of many factors can cause water retention, urine volume is reduced, which may cause the FGF-21 clearance rate to be decreased. On the contrary, hyperthyroidism can make the body's metabolism strong, and its basal metabolism is increased obviously. The acceleration of protein decomposition and lipid oxidation and decomposition may be the main reason for the decrease of serum FGF-21 in patients. In this study, serum free thyroxine TSH, FT3 and FT4 level can be compared. The result showed that hypothyroidism group in serum TSH is significantly higher than that in the control group, FT3 and FT4 is significantly lower than that in the control group; serum TSH in patients with hyperthyroidism group is significantly lower than that in the control group, free thyroxine FT3 and FT4 is significantly higher than that in the control group, which showed that patients with abnormal thyroid function of thyroid gland can make the function of the serum TSH secretion disordered, having effect on the uptake of iodine and thyroglobulin (TG) iodide, affecting the synthesis of T4 and T3. The study found that positive hypothyroidism patients plasma FGF-21 levels is positively correlated with age, TSH, LDL 
and other related parameters, the research shows that the level of plasma FGF-21 in patients with hypothyroidism is increased which may be involved in hormone metabolism, as well as the regulation of lipid, resulting in one of the risk factors of thyroid dysfunction.

In conclusion, through this research, the expression level and its correlation of serum FGF-21serum FGF-21 in patients with hypothyroidism as well as biochemical index is analyzed, so as to find out that the expression level of serum FGF-21 in patients with hypothyroidism is higher, which can be involved in the occurrence and development of diseases; serum TC, TG and LDL is increased, it can be the common reasons to cause dyslipidemia, it also can provide valuable clues for the research of the development as well as the treatment of hypothyroidism, which is needed to be confirmed in the further study.

\section{Acknowledgement}

National Natural Science Foundation, Fund Number: 81273421, Innovation and Entrepreneurship Program for College Students.

\section{Reference}

[1] Fan JG, Farrell GC. Epidemiology of non-alcoholic fatty liver disease in China[J]. J Hepatol, 2009, 50(1): 204-210.

[2] Stein S, Bachmann A, Lössner U, et al. Serum levels of the adipokine FGF21 depend on renal function, Diabetes Care[J]. 2009, 32(1):126-128.

[3] Lee Y, Park YJ, Ahn HY, et al.Plasma FGF21 levels are increased in patients with hypothy -roidism independently of lipid profile[J]. Endocr J, 2013, 60(8):977-83.

[4] Cheng X, Zhu B, Jiang F, et al. Serum FGF-21 levels in type 2 diabetic patients[J], Endocr Res, 2011, 36(4):142-148.

[5] Moyers JS, Shiyanova TL, Mehrbod F, et al. Molecular determinants of FGF-21 activity-synergy and cross -talk with PPARgamma signaling[J]. J Cell Physiol, 2007, 210(1): $1-6$.

[6] Semba RD, Sun K, Egan JM, et al. Relationship of serum fibroblast growth factor 21 with abnormal glucose metabolism and insulin resistance.The Baltimore longitudinal study of Aging[J]. J Clin Endocrinol Metab, 2012 ,97(4):1375-1382.

[7] Mason RL, Hunt HM, Hurxthal L. Blood Cholesterol Values in Hyperthyroidism and Hypothyroidism Their Significance[J]. New England Journal of Medicine,1930, 203( 26): 1273-1278.

[8] Sinha RA,You SH,Zhou J, et al.Thyroid hormone stimulates hepatic lipid catabolism via activation of autophagy[J]. J Clin Invest, 2012,122( 7) : 2428-2438.

[9] Adams AC, Astapova I, Fisher FM, et al. Thyroid hormone regulates hepatic expression of fibroblast growth factor 21 in a PPARalpha-dependentmanner[J]. J Biol Chem, 2010, 285(19):14078-14082. 